\title{
Bifosfonatos asociado a osteonecrosis de los maxilares. Revisión de la literatura
}

\author{
Jaimes $M^{*}$, Oliveira GR**, Olate $S^{*}, * * *$, Albergaria Barbosa JR de****
}

\section{RESUMEN}

Los bifosfonatos son una familia de drogas usadas comúnmente en el tratamiento de alteraciones óseas causadas principalmente por tumores malignos y osteoporosis. En el año 2003 fue relatado el primer caso de osteonecrosis de los maxilares asociado al uso de bifosfonatos. Esta condición patológica presenta un mal pronóstico y una incidencia creciente. El objetivo de este artículo es orientar a los cirujanos dentistas sobre los factores causales y formas de tratamiento de dicha alteración.

Palabras clave: Bifosfonatos, osteonecrosis, cáncer, osteomielitis, metástasis ósea.

\section{SUMMARY}

The bisphosphonates are a family of drugs commonly used for treatment of bone changes caused mainly by malignant neoplasias and osteoporosis. In 2003 was described the first bisphosphonate related osteonecrosis of the jaws, whose causes are still unknown. This pathologic condition has a bad prognosis and an increasing incidence. The aim of this work is to advice, is about the etiologic factors and treatment options for this alteration.

Key words: Bisphosphonate, osteonecrosis, cancer, osteomielitis, bone metastasis.

Fecha de recepción: Septiembre 2007.

Aceptado para publicación: Diciembre 2007.

* Alumno de Maestría en Cirugía y Traumatología Buco-Maxilo-Facial. Facultad de Odontología de Piracicaba. Universidad Estadual de Campinas - UNICAMP, Brasil.

** Alumno de Doctorado en Cirugía y Traumatología Buco-Maxilo-Facial. Facultad de Odontología de Piracicaba. Universidad Estadual de Campinas - UNICAMP, Brasil.

*** Profesor, Departamento de Odontología Integral, Facultad de Medicina. Universidad de La Frontera, Chile.

**** Profesor Titular, Área de Cirugía y Traumatología Buco-Maxilo-Facial, Departamento de Diagnostico Oral. Facultad de Odontología de Piracicaba, Universidad Estadual de Campinas - UNICAMP, Brasil.

Jaimes M, Oliveira GR, Olate S, Albergaria Barbosa JR de. Bifosfonatos asociado a osteonecrosis de los maxilares. Revisión de la literatura. Av. Odontoestomatol 2008; 24 (3): 219-226.

\section{INTRODUCCIÓN}

Hace una década, los bifosfonatos se introdujeron como una alternativa a las terapias de reemplazo de hormonas para el tratamiento de osteoporosis y para tratar tumores osteolíticos. Más recientemente, ha sido evidente que estas drogas específicamente pamidronato y zoledronato podrían llevar a exposiciones óseas dolorosas llamada osteonecrosis de los maxilares (ONM), estableciendo una fuerte relación entre ellas (1). 
A pesar de que esta lesión puede manifestarse espontáneamente, se han descrito una serie de factores de riesgo para la aparición, como antecedentes de radioterapia, uso de corticoides o el tratamiento con talidomida y como factor desencadenante en la mayoría de los casos aparece asociada a procedimientos odontológicos. El riesgo de osteonecrosis puede aumentar con las extracciones dentales y la mala higiene bucal, de tal manera que al exponerse el hueso a la flora, se infecta produciendo dolor, inflamación, infección con supuración y, finalmente necrosis ósea.

Esta lesión no tiene un tratamiento eficaz por lo que ocasiona una gran morbilidad a los pacientes. Siempre que sea posible se deberán evitar las extracciones dentales y toda cirugía oral electiva en pacientes que hacen uso de estas drogas. Si la cirugía es esencial el paciente debe ser informado sobre los riesgos.

\section{REVISIÓN DE LA LITERATURA}

Los bifosfonatos son potentes inhibidores de los osteoclastos que por interacción con estas células producen una marcada disminución de la reabsorción ósea. Se utilizan habitualmente en los pacientes con cáncer avanzado, metástasis óseas e hipercalcemia maligna, entre otras $(1,2)$.

En estos individuos con ciertas patologías que afectan el sistema óseo y que están en tratamiento odontológico o por comenzarlo, conociendo o ignorando su condición, el proceso de aposición y reabsorción ósea puede estar alterado (3), por ello es importante conocer el efecto que puede tener el uso de bifosfonatos en el componente buco-maxilofacial ya que estos fármacos son los que se prescriben para dichas patologías.

A pesar de que estas drogas fueron sintetizadas en el siglo XIX, el conocimiento sobre sus características biológicas data desde la década de 1960. En el pasado, estos componentes fueron utilizados para múltiples propósitos industriales, como agente antisedimentación y para prevenir la formación de carbonato de calcio.

En el año de 1865, el Prof. Fleisch y colaboradores observaron que el pirofosfato llevaba cristales de fos- fato para el calcio impidiendo la formación de cristales y disolución in Vitro. En colaboración con el Dr. M. D. Francis y Dr. R.G.G Rusell, Prof. Fleisch y colaboradores mostraron que los análogos de los pirofosfatos, bifosfonatos llamados difosfonatos, interactuaban de forma similar al fosfato de calcio in Vitro, inhibiendo tanto la mineralización como la reabsorción ósea animal (4).

En 1969 fue publicado por primera vez sobre los bifosfonatos en el Sciencie and Nature. Veinte años más tarde estos elementos fueron utilizados en las drogas para disfunciones óseas (5).

Los bifosfonatos presentan en su composición química dos átomos de fósforo unidos a un átomo de carbono (P-C-P). Esta estructura les confiere una resistencia a la hidrólisis enzimática y les permite fijarse con gran avidez a la superficie de los cristales de hidroxiapatita con una rápida y eficiente unión del fármaco a la superficie mineral ósea. Su acción primordial consiste en inhibir la resorción ósea mediante la supresión de la actividad de los osteoclastos (6).

Berenson (7) (1998) observó que el uso de pamidronato mejoraba la supervivencia y disminuía las complicaciones óseas. Este y otros estudios llevaron a propugnar el uso de estas drogas de forma indefinida $(8,9)$.

En el año 2003 fue relatado por primera vez la relación existente entre el bifosfonatos y la ONM (10). Desde entonces se han reportado numerosos casos que describen este nuevo cuadro clínico que acomete a los maxilares (1).

Marx y Stern (11) relataron que los pacientes con mieloma múltiple que reciben pamidronate podrían desarrollar necrosis avascular de los maxilares.

Diversas publicaciones informan sobre pacientes que procuraron tratamiento para necrosis ósea intraoral que ocurrió espontáneamente después de extracciones dentales o trauma oral $(11,12)$. Estos pacientes presentaron una historia de malignidad primaria, que incluye mieloma múltiple, carcinoma de mama y próstata, siendo tratados con pamidronate intravenoso o el ácido zoledrónico. 
Otros estudios sugieren que la dosis excesiva de bifosfonatos puede comprometer la calidad y densidad ósea (14). Este nuevo tipo de osteonecrosis de los maxilares es generalmente asociado a pacientes que presentan cáncer con metástasis ósea que son tratados con bisfosfonatos y aquellos pacientes que reciben tratamiento para la prevención de osteoporosis $(15,16)$.

Wang et al (10) describe el caso de 3 pacientes del sexo femenino que recibieron quimioterapia para el tratamiento de cáncer de mama con metástasis, 2 pacientes desarrollaron necrosis óseas subsecuentes a procedimientos dentales y 1 paciente desarrollo espontáneamente osteonecrosis en la maxilar. Este autor relata que la osteonecrosis fue causada por la quimioterapia con docetaxel y paclitaxel, sin embargo en un reporte posterior sobre la relación de los bifosfonatos con la ONM los autores revelan que 3 pacientes fueron tratados con bifosfonato (12).

Un estudio informo sobre la ONM en 18 pacientes, posterior al tratamiento con pamidronate o el ácido zoledrónico por un periodo mínimo de 25 meses, 17 pacientes presentaban cáncer con metástasis ósea y 1 presentaba osteopenia. Del sexo femenino fueron reportados 14 casos y 4 del sexo masculino con una edad promedio total de 62 años. Las características clínicas de la ONM en la mayoría de los casos incluyeron infección y necrosis ósea mandibular asociados a extracciones dentales, infección y trauma. Dos pacientes desarrollaron osteonecrosis espontáneamente, sin evidencia clínica o radiográfica que pudiera sugerir alguna patología relacionada (2).

Ruggiero et al (2004) (13) reporta una revisión de 63 pacientes que habían recibido tratamiento con bifosfonatos intravenoso y pacientes que estaban en tratamiento crónico con bifosfonatos oral. De estos 63 pacientes, 28 presentaban mieloma múltiple; 20 pacientes, con diagnóstico de cáncer de mama; 3 presentaban cáncer de pulmón; 3, con cáncer de próstata; 1 presentaba plasmacitoma y 1 paciente, con diagnóstico de leucemia. Otros 7 pacientes, con diagnóstico de osteoporosis, no recibían tratamiento de quimioterapia.

En este estudio, el autor reporta que 39 pacientes presentaron osteonecrosis de la mandíbula y 23 en la maxilar siendo que un paciente presentó osteonecrosis tanto en maxilar como en mandíbula. Otro dato importante es que 54 pacientes relataron historia de extracción dental reciente en el lugar de la necrosis mientras que 9 pacientes presentaron exposición ósea espontánea.

Bagan et al (2005) (17) publicó una serie de 10 pacientes que presentaron osteonecrosis mandibular posterior al tratamiento de cáncer con quimioterapia. Estos autores relataron que el $50 \%$ de los casos de osteonecrosis fue localizado en la maxilar. En la mayoría de los casos esta lesión fue precedida por extracciones dentales.

Durie et al (2005) (18) publicó los resultados de una revisión realizada por la Fundación Internacional Mieloma, sobre los factores de riesgo en la osteonecrosis de los maxilares. Un total de 1.203 pacientes (904 con mieloma múltiple y 299 con cáncer de mama) participaron en la revisión. Estos autores determinaron que 62 pacientes con mieloma múltiple presentaban osteonecrosis de los maxilares mientras que 54 pacientes con indicio de presentar la lesión. En los pacientes con cáncer de mama 13 pacientes presentaban ONM y 23 pacientes con sospecha de presentar la lesión. Corticosteroides y talidomida estaban asociados con el desarrollo de ONM. El 81\% de los casos de pacientes con manifestación de la lesión presentaba historia de problemas dentales. Este estudio reporto que el $10 \%$ de los pacientes que recibieron ácido zoledrónico y el $4 \%$ de los pacientes que recibieron pamidronate desarrollaron ONM.

Un estudio retrospectivo de 4.000 pacientes que recibían tratamiento con bifosfonatos intravenoso, identificó 33 casos de osteonecrosis mandibular (0,83\% total de incidencia); 14 pacientes, con diagnóstico de cáncer de mama (incidencia de 1,2\%) y 15 pacientes, con tratamiento para mieloma múltiple (incidencia de 2,8) (19).

Por su parte Otolina en el (2005) (20) realiza una revisión retrospectiva de 5 pacientes que hacían uso de bifosfonatos y que desarrollaron ONM. Todos estos pacientes presentan historia previa de extracción dental. Es importante que los oncologistas y los odontólogos conozcan sobre los posibles efectos que pueden producir los bisfosfonatos, este autor reco- 
mienda realizar todo procedimiento de cirugía oral menor antes de iniciar el uso de estas drogas.

Chiandussi et al (2006) (21), relata sobre la importancia de realizar un diagnóstico clínico y por imágenes en aquellos pacientes que presentan ONM asociado a bifosfonatos. Identificar precozmente la lesión permitirá establecer un tratamiento adecuado evitando mayores consecuencias. Estos autores realizaron un estudio en 11 pacientes que presentaban ONM asociado a bifosfonatos y los cuales fueron sometidos a exámenes de imagen (radiografía convencional , tomografía computadorizada, resonancia magnética y ${ }^{99 \mathrm{~m}}$ Tc-MDP 3-phase con la finalidad de describir la lesión. Como resultado, la radiografía convencional y la tomografía computadorizada exhiben lesiones osteolíticas a nivel del hueso cortical, en la resonancia magnética se observa un aspecto característico de osteonecrosis y edema en el tejido blando. La tomografía computadorizada y la resonancia magnética fueron muy útiles para definir la extensión de la lesión.

Kumar et al (2007) (22), reportan 3 casos de pacientes con ONM, describen los resultados de tratamientos y morbilidad de los mismos. Estos casos presentan estudios de imágenes comúnmente usados en la odontología: radiografía panorámica y tomografía computadorizada. El uso de los exámenes de imágenes puede permitir detectar precozmente esta lesión estableciéndose así un tratamiento adecuado. Estos autores señalan sobre el peligro de realizar procedimientos dentales invasivos en pacientes que reciben bifosfonatos.

La Asociación Americana de Cirugía Oral y Maxilofacial (2007) (23), emite un escrito en relación a bifosfonatos y osteonecrosis de los maxilares. El propósito de esta publicación es fijar una posición y proporcionar directrices al respecto. Como primer punto la AACOM describe sobre el riesgo que existe en un paciente que hace uso de bifosfonatos de desarrollar ONM. Riesgos y beneficios de los bifosfonatos deben ser tomados en consideración al momento de decidir sobre el tratamiento que será llevado cabo. Como segundo punto, directriz de los clínicos con respecto al diagnóstico diferencial de la ONM en pacientes con historia de tratamiento de bifosfonatos por vía intravenosa o vía oral. Y por ultimo, seña- lan sobre las medidas preventivas que deben ser tomadas en cuentas al momento de trata un paciente que haga uso de estas drogas. El tratamiento odontológico de los pacientes que están en tratamiento con bifosfonatos o que iniciaran el uso de los mismos permitirá actuar de manera preventiva. Si el paciente manifiesta la lesión el mismo sera tratado dependiendo del estado actual de la enfermedad.

\section{PREVENCIÓN Y MANEJO DE LA OSTEONECROSIS DE LOS MAXILARES}

Han sido identificados factores de riesgo para la ONM y también se ha especulado que la irrigación con microvasculatura y la presencia microbiana en el medio ambiente oral podrían favorecer el curso de la enfermedad (24).

Recientemente dos publicaciones fueron realizadas en relación a la prevención y manejo de pacientes con ONM (13). Una de las publicaciones contiene directrices de la Academia Americana de Medicina Oral y la otra publicación fue escrita por un grupo de Odontólogos y oncologistas. Ambas publicaciones presentan similar abordaje para aquellos pacientes con ONM. Estas estrategias no están basadas en estudios clínicos, son usadas en pacientes con osteorradionecrosis.

\section{DIRECTRICES DESARROLLADAS PARA TRES GRUPOS DISTINTOS DE PACIENTES}

\section{Medidas preventivas para pacientes próximos} a iniciar tratamiento con bifosfonatos:

- Elementos dentales con mal pronóstico deben ser extraídos.

- Cualquier procedimiento dental quirúrgico debe ser finalizado antes del tratamiento con bifosfonatos.

- Estos pacientes deben ser examinados por el odontólogo, clínica y radiográficamente, enfatizando la necesidad de mantener una buena higiene bucal.

2. Medidas preventivas para pacientes que están en tratamiento con bifosfonatos:

- Mantener un control odontológico de 3 a 6 meses. 
- Limpieza dental de rutina debe ser realizada con cuidado, evitando heridas en el tejido blando.

- Dentaduras removibles deben ser chequeadas por su potencial de inducir trauma.

- El tratamiento endodóntico es preferido antes la extracción dental.

- Implantes dentales deben ser evitados.

- Si la cirugía es necesaria, debe considerarse la prescripción de antibiótico prequirúrgico y postquirúrgico por un periodo mínimo de 10 días.

- Cicatrización por primera intención de todas las heridas orales incluyendo sitios de extracción debe ser intentado.

\section{Tratamiento de pacientes con osteonecrosis de los maxilares:}

- Debido a la cicatrización tardía en pacientes con ONM, los accesos quirúrgicos no son recomendados.

- Un desbridamiento mínimo de la lesión debe ser realizado, para eliminar los bordes óseos agudos.

- Dispositivos removibles intrabucales son recomendados para proteger a la lesión de futuros traumas.

- Enjuagues con digluconato de clorexidina al $0,12 \%$ es recomendado en todo los casos.

El uso de antibióticos para tratar lesiones causadas por ONM ha tenido resultados ambiguos sin embargo este va a depender del criterio clínico. La elección del antibiótico será determinada preferiblemente por el estudio de cultura y la prueba de sensibilidad de la lesión. Se ha sugerido que la combinación de amoxicilina y metronidazol puede dar buenos resultados $(12,13)$.

Pacientes con ONM pueden presentar infección fúngica, en estos casos debe ser comprobado a través de la prueba de cultura y debe ser tratado con nistatina oral o micostatina en pastillas. Microorganismos, aerobios mixtos y anaerobios han sido asociados también a esta lesión (24). El constante proceso de remodelación ósea junto a las demandas sufridas por los tejidos óseos maxilares y mandibulares permiten que el metabolismo óseo sea eficiente y constante, lo cual permitiría también un mayor desarrollo de la ONM. (25) En pacientes en los cuales el tratamiento conservador no ha sido eficaz se debe planificar una intervención quirúrgica para eliminar el tejido óseo necrótico (26). La terapia con oxígeno hiperbárico es empleado para la prevención y tratamiento de osteorradionecrosis del componente maxilofacial. En pacientes con ONM el uso de esta es bastante controversial. A pesar de no existir relatos sobre el éxito de la terapia de oxígeno hiperbárico en pacientes con ONM ya este ha sido empleado. Cuando sea posible se deberán evitar las extracciones dentales y toda cirugía oral electiva en pacientes que hacen uso de bifosfonatos. Si la cirugía es esencial el paciente debe ser informado sobre los posibles riesgos.

\section{DISCUSIÓN}

Los bifosfonatos son potentes inhibidores de los osteoclastos, con una vida media de años, estas drogas son usadas principalmente para el tratamiento de mieloma múltiple y en metástasis óseas de tumores sólidos como el cáncer de mama, estas drogas reducen los eventos óseos significativamente $(8,9)$. Aun no se ha establecido una relación causa efecto de estas drogas sobre la ONM, sin embargo la incidencia en tiempos anteriores en relación al uso de bifosfonatos era muy baja, $(27,28)$ siendo que hoy día alcanza $10 \%$ o más en las patologías mencionadas tratadas con zoledronato y algo menos con otros (18).

Como explicación de la frecuente localización en los maxilares, se ha resaltado el estrés fisiológico a que son sometidas dichas áreas, únicas en exponer hueso al medio externo. Este estrés aumenta por procesos patológicos dentales y periodontales: abscesos, iatrogenia (tratamiento endodóntico, extracciones dentales y otros), que exigen aumentar la velocidad de recambio o remodelación ósea, la que se encuentra bloqueada por dichas drogas $(28,29)$. A esto se agrega el efecto antiangiogénico de los bifosfonatos, que provocan disminución del factor de crecimiento de endotelios, $(27,18)$ posiblemente explicando la mayor frecuencia de compromiso de la mandíbula (63-80\%), hueso de menor irrigación en relación al maxilar superior (14-38\%) $(11,26)$. La mayoría de los casos de osteonecrosis se presentan posterior a una extracción dental (78\%), infección u otro proceso y 
sólo una minoría (14\%) aparece espontáneamente. Sólo 5,5\% se presentan en ambos maxilares $(11,27)$.

Pacientes con factores de riesgo mencionados, se han comunicado las siguientes recomendaciones en la prevención: revisión dental previa al inicio de los bifosfonatos, seguimiento y tratamiento de cualquier patología dentomaxilar, extracción de restos radiculares, tratamiento de la enfermedad periodontal, obturación de caries, con el mismo criterio recomendado para pacientes candidatos a radioterapia (28). Higiene dental con antisépticos locales durante la terapia (14). Realizar imágenes radiológicas ante cualquier síntoma local $(7,3)$. Evitar cirugía dental (extracciones, implantes, debridaciones, etc) durante la terapia $(11,9,30)$. Suspender bifosfonatos 3 meses antes de procedimientos dentales que se consideren imprescindibles durante la terapia (31).

En el manejo de la osteonecrosis establecida, las recomendaciones son: suspensión del bifosfonato, higienización oral con clorhexidina $0,12 \%$ en áreas óseas expuestas y especialmente en la zona de inserción mucoperióstica, que es propicia a la colonización bacteriana. Extracción de dientes desvitalizados comprometidos en el área de osteonecrosis. En caso de molestia lingual por contacto con áreas óseas o en caso de infección secundaria persistente, puede practicarse ostectomías limitadas. Cobertura antibiótica para evitar infección secundaria. El uso de oxigenoterapia en cámara hiperbárica, para mejorar la oxigenación local, no se ha demostrado uniformemente eficaz (13).

\section{CONCLUSIÓN}

La ONM ha sido recientemente asociada al uso prolongado de bifosfonatos. La importante reducción de los eventos óseos en mieloma y cáncer de mama metastático que aportan, los hace necesarios. Estas drogas deben ser usadas con precaución, especialmente en aspectos preventivos. Conocer sobre las medidas preventivas recomendadas para aquellos pacientes que serán sometidos a tratamientos odontológicos y que iniciarán o están en tratamiento con bifosfonatos, o en el último de los casos hayan desarrollado ONM, nos permitirá decidir sobre la ejecución o no de ciertos tratamientos odontológicos.

\section{BIBLIOGRAFÍA}

1. Merigo E, Manfredi M, Meleti M, Guidotti R, Ripasarti A, Zanzucchi E, D'Aleo P, Corradi D, Corcione L, Sesenna E, Ferrari S, Poli T, Bonaninil M, Vescovi P. Bone necrosis of the jaws associated with bisphosphonate treatment: a report of twenty-nine cases. Acta Biomed 2006; 77:109-17.

2. Migliorati CA, Schubert MM, Peterson DE, Seneda LM et al. Bisphosphonate-associated osteonecrosis of mandibular and maxillary bone. Cancer 2005; 104:83-93.

3. Haruyama N, Igarashi K, Saeki S, Otsuya-Isoya M, Shinoda H, Mitani H. Estrous-cycle-dependent variation in orthodontic tooth movement. J Dent Res 2002; 6:406-10.

4. Fleish H, Russell RG, Francis MD. Diphosphonates inhibit hydroxyapatite dissolution in vitro and bone resorption in tissue culture and in vivo. Science 1969;165:1262-4.

5. Fleish H, Russell RG, Simpsom B, Muhlbauer RC. Prevention by diphosphonate of immobilization osteoporosis in rats. Nature 1969; 223:211-2.

6. Ellemann K, Sjogren P, Banning AM, Jensen D. Trial of intravenous lidocaine on painful neuropathy in cancer patients. Clin J Pain. 1989; 5:291-294.

7. Berenson JR, Lichtenstein A, Porter L, Dimopoulos MA, Bordoni R, George S ET AL. Long-term pamidronate treatment of advanced multiple myeloma patients reduces skeletal events. Myeloma Aredia Study Group.I Journal Clinical Oncology 1998;16:593-02.

8. Berenson JR, Lichtenstein A, Porter L et al. Efficacy of pamidronate in reducing skeletal events in patients with advanced multiple myeloma. N Engl J Med 1996;334:488-93.

9. Berenson JR, Rosen LS, Howell A et al. Zoledronic acid reduces skeletal-related events in patients with osteolytic metastases Cancer 2001;91:1191-200. 
10. Wang J, Goodger NM, Pogrel MA. Osteonecrosis of the jaws associated with cancer chemotherapy. J Oral Maxillofac Surg 2003;61:1104-7.

11. Marx RE, Stern D. Oral and maxillo-facial pathology: a rationale for diagnosis and treatment. 1st ed, chapter 2. Carol Stream, IL: Quintessence 2003;2:36-8.

12. Migliorati CA. Bisphosphonates and oral cavity avascular necrosis of bone. J Clin Oncol 2003; 21:4253-4.

13. Ruggiero SL, Mehrotra B, Rosenberg TJ, Engroff SL. Osteonecrosis of the jaws associated with the use of bisphosphonates:a review of 63 cases. J Oral Maxillofac Surg 2004;62:527-34.

14. Pogrel MA. Bisphosphonates and bone necrosis. J Oral Maxillofac Surg. 2004;62:391-92.

15. Whyte MP, Wenkert D, Clements KL, McAlister WH, Mumm S. Bisphosphonate-induced osteopetrosis [brief report]. N Engl J Med. 2003;349:457-63.

16. Marini JR. Do bisphosphonates make children's bones better or brittle [editorial]? N Engl J Med. 2003;349:423-6.

17. Bagan JV, Murillo J, Jimenez Y, et al: Avascular jaw osteonecrosis in association with cancer chemotherapy: Series of 10 cases. J Oral Pathol Med 2005;34:120-3.

18. Durie BGM, Katz M, Crowley J: Osteonecrosis of the jaw and bisphosphonates. N Engl J Med 2005;353:99-100.

19. Hoff AO,Toth B, Altundag K, et al.Osteonecrosis of the jaw in patinents receiving intravenous bisphosphona tetherapy. Pesented at: 27 th Annual Meeting of the American Society for boné and Mineral Research; September 2005; Nashvile, Tenn.

20. Oltolina A, Achilli A, Lodi G, Demarosi F, Sardella A. Osteonecrosis of the jaws in patients treated with bisphosphonates. Review of the literature and the Milan experience. Minerva Stomatol. 2005; 54:441-8.
21. Chiandussi S, Biasotto M, Dore F, Cavalli F, Cova MA, Di Lenarda R. Clinical and diagnostic imaging of bisphosphonate-associated osteonecrosis of the jaws. Dentomaxillofac Radiol. 2006;35:236-43.

22. Kumar V, Pass B, Guttenberg SA, Ludlow J, Emery RW, Tyndall DA, Padilla RJ. Bisphosphonate-related osteonecrosis of the jaws: A report of three cases demonstrating variability in outcomes and morbidity. J Am Dent Assoc. 2007; 138:602-9.

23. Advisory Task Force on Bisphosphonate-Related Ostenonecrosis of the Jaws. American Association of Oral and Maxillofacial Surgeons position paper on bisphosphonate-related osteonecrosis of the jaws. J Oral Maxillofac Surg. 2007; 65:369-76.

24. Mehrotra B, Ruggiero S. Bisphosphonate complications including osteonecrosis of the jaw. Hematology Am Soc Hematol Educ Program. 2006;1:356-60.

25. Gutta R, Louis PJ. Bisphosphonates and osteonecrosis of the jaws: Science and rationale. Oral Surg Oral Med Oral Pathol Oral Radiol Endod. 2007;104:186-93.

26. Jiménez Soriano Y, Bagan JV. Los bifosfonatos, nueva causa de osteonecrosis maxilar por fármacos: situación actual. Med Oral Patol Oral Cir Bucal 2005; 10 Suppl2: E88-91.

27. Vitte C, Fleisch H, Guentes HL. Bisphosphonates induce osteoblasts to secrete an inhibitor of osteoclastic mediated resorption. Endocrinology 1996;137: 2324-33.

28. Sook-Bin W, Hande K, Richardson PG. (letter). N Eng J Med 2005;353:100.

29. Santini D, Vincenzi B, Avvisati G, Dicuonzo G, Battistoni F, Gavasci M et al. Pamidronate induces modifications of circulating angiogenic factors in cancer patients. Clin Cancer Res 2002; 8:1080-4. 
30. Tarassoff P, Yong-jiang Hei. (letter) Reply from Novartis. N Engl J Med 2005; 353: 101 2.

31. Thakkar SG, Isada C, Smith J, Karam MA, Reed J, Tomford JW et al. Bisphosphonate therapy and increased incidence of mandibular/maxillary osteomyelitis. Haematologica Hem Journal 2005; $1: 191$.

\section{CORRESPONDENCIA}

Prof. Dr. José Ricardo de Albergaria Barbosa

Departamento de Diagnóstico Oral.

Área de Cirugía y Traumatología Buco-Maxilo-facial Avenida Limeira, 901, Bairro: Areão.

CEP: 13414-903. Piracicaba-SP

Fone: (55) 19 - 2106-5377.

e-mail:barbosa@fop.unicamp.br 